\title{
QUANTITATIVE SPECTRAL CLASSIFICATION IN THE BCD SYSTEM FOR LMC SUPERGIANTS
}

\author{
L. DIVAN \\ Institut d'Astrophysique de Paris and European Southern Observatory, La Silla, Chile
}

\begin{abstract}
In December 1970 and January 1971, spectra of 13 LMC supergiants have been obtained with the Chalonge spectrograph attached to the Cassegrain focus of the $152 \mathrm{~cm}$ ESO reflector at La Silla. The spectrophotometric parameters $\lambda_{1}, D, \varphi_{b}$ and $\varphi_{u v}$ were measured and the first results deal with (a) spectral classification and (b) the distance modulus of the LMC.
\end{abstract}

\section{Spectral Classification}

The $\lambda_{1} D$ diagram has been calibrated in absolute magnitudes by means of galactic supergiants belonging to clusters. The result (curves of equal absolute magnitude from $M=0$ to $M=-8$ ) was presented at the IAU meeting in Brighton (1970).

If the LMC distance modulus is as large as generally thought, the Cloud contains supergiants 1 or 2 magnitudes brighter than the brightest ones known in our Galaxy. Thus, the program stars in the LMC have been divided into two groups:

(I) the brightest B and A type stars,

(II) a few B and A type stars about $1.5 \mathrm{mag}$. fainter than those of the first group.

The results are:

(1) The two groups of stars are well separated in the $\lambda_{1} D$ plane: the second group lies in the region of the brightest Ia galactic supergiants, between the curves $M=-7$ and $M=-8$, and the first group well outside the curve $M=-8$ in the direction of highter luminosities.

(2) The spectral types of all these stars, deduced from their position in the $\lambda_{1} D$ diagram, are generally in very good agreement with the spectral types determined by Ardeberg et al. (1972).

\section{Distance Modulus of the LMC}

Making the assumption that the LMC stars do not differ fundamentally from the galactic ones and that the curves of equal absolute magnitudes in the $\lambda_{1} D$ plane are still valid for them, the $M$ values for stars of the second group (stars between the curves, $M=-7$ and $M=-8$ ) result from the position on the $\lambda_{1} D$ diagram. The $V_{0}$ magnitudes were obtained from the $V$ magnitudes corrected for the interstellar absorption $A_{v}$; the value of $A_{v}$, which is always small, was calculated from the excess in blue gradient, $\varphi_{b}-\varphi_{0 b}$, and assuming a normal reddening law. The mean distance modulus $V_{0}-M$ given by five different stars (with only one spectrum for each) is $18.1 \pm 0.5$. This value is smaller than those generally derived and more observations are desirable. The absolute magnitudes of the first group of stars (the brightest LMC supergiants) calculated from this distance modulus, lie between -9 and -9.5 , and the curve of equal absolute magnitude $M=-9$ could be added to the $\lambda_{1} D$ diagram. 
Finally the case of the $F$ type object $\mathrm{G} 296$ has been discussed: with a line spectrum F6Ia, it has the blue gradient $\varphi_{b}$ of a F0 or F2 star and the $\lambda_{1} D$ type is about F9Ia; up to now, nothing similar has been observed in our Galaxy.

\section{Reference}

Ardeberg, A., Brunet, J.-P., Maurice, E., and Prévot, L.: 1972, Astron. Astrophys. Suppl. Ser. 6, 249. 